\title{
Meeting report \\ 42nd Annual Meeting of the American Society for Cell Biology, San Francisco, California, USA, 14-18 December 2002
}

\author{
Paraic A Kenny and Aylin Rizki
}

Life Sciences Division, Lawrence Berkeley National Laboratory, Berkeley, California, USA

Corresponding author: Aylin Rizki (e-mail: arizki@lbl.gov)

Published: 11 March 2003

Breast Cancer Res 2003, 5:147-153 (DOI 10.1186/bcr590)

(C) 2003 BioMed Central Ltd (Print ISSN 1465-5411; Online ISSN 1465-542X)

\begin{abstract}
The Annual Meeting of the American Society for Cell Biology (ASCB) is a diverse conference covering all topics in cell biology. While all of the basic biology presented at this meeting may potentially contribute to breast cancer research, there were a significant number of presentations and posters directly pertinent to this field. Here we have summarized the research that is of greatest immediate relevance to breast cancer, with particular emphasis on mammary gland development and tumorigenesis in vivo, three-dimensional in vitro models of mammary morphogenesis, alterations of signal transduction pathways in breast cancer, and global studies in expression profiling and drug screening.
\end{abstract}

Keywords: basement membrane, breast cancer, matrix metalloproteinase, metastasis, signal transduction

\section{Introduction}

During a rainy, windswept San Francisco week in midDecember, over 8000 scientists found shelter at the $42 \mathrm{nd}$ annual meeting of the American Society for Cell Biology (ASCB) in the Moscone Convention Center. Attendees were spoiled for choice, with a diverse array of high-quality research ranging from bacterial environmental sensing to the modeling of evolution. The talks ranged from excellent plenary lectures discussing some of the most important and exciting issues facing cell biologists today, to smaller, more intimate minisymposia that focused on single topics and allowed for lively discussions from a larger number of contributors. The meeting opened on Saturday afternoon with 24 parallel special-interest-group meetings followed by the four keynote lectures. There were 8 major symposia of three lectures each over the succeeding four mornings, and a staggering total of 232 minisymposium talks in the afternoons. These minisymposia represented an embarrassment of riches and it was often difficult to decide which sessions to attend. Additionally, there were several outstanding lectures from this year's ASCB award- winners, Avram Hershko, Alexander Varshavsky, Kathleen Collins, Frank Talamantes, Benjamin Cravatt, Eric Wieschaus, Natasha Raikhel, and Clare Waterman-Storer. Over 3000 posters were presented, giving a large proportion of the attendees the opportunity to communicate their research to a wide and appreciative audience. This year's ASCB social took place in the outstanding San Francisco Museum of Modern Art, allowing the delegates to let their hair down, mingle, and contemplate an entirely different set of abstractions from those on display across the street at the Moscone Center.

It would be impossible in a brief report to attempt to communicate the vast depth and breadth of research presented during this meeting, so we have elected to concentrate our efforts on discussing some of the work of most relevance to breast cancer research. The subheadings used do not directly reflect the sessions of the ASCB meeting but were chosen to provide a meaningful framework for the presentation of this research. growth factor; EGFR = epidermal-growth-factor receptor; ER- $\alpha=$ estrogen receptor alpha; HMECs = human mammary epithelial cells; IL $=$ interleukin; LAP = liver-enriched transcriptional activator protein; LIF = leukemia inhibitory factor; LIP = liver-enriched inhibitory protein; MAPK = mitogen-activated protein kinase; MMP = matrix metalloproteinase; MMTV = mouse mammary tumor virus; -MMP = membrane-type matrix metalloproteinase; $\mathrm{PI} 3 \mathrm{~K}=$ phosphoinositide 3-kinase; siRNA = small interfering ribonucleic acid; TNF- $\alpha=$ tumor necrosis factor alpha. 


\section{Mammary gland development and tumorigenesis in vivo}

Many new in vivo mouse models of mammary gland development were presented. Roles of matrix metalloproteineases and other extracellularly presented or secreted molecules such as epimorphin, cytokines, and secreted glycoproteins, as well as multiple transcription factors, were investigated. In addition, two reports on the stem cell compartment of the mouse mammary gland were presented.

\section{Cytokines}

Argentinean scientists, led by Carolina Schere-Levy (Experimental Medicine, Academia Nacional de Medicina, Buenos Aires, Argentina) reported their studies on the expression of leukemia inhibitory factor (LIF) during the developmental stages of the mouse mammary gland. LIF is a glycoprotein and a cytokine that regulates growth and differentiation. She and her colleagues in Edith C Kordon's group showed for the first time that LIF has a role in the involution stage of the mammary gland cycle. They showed that expression of LIF is increased significantly during the early stages of involution. Mechanistically, LIF expression is induced by milk stasis. Furthermore, ectopic expression of LIF results in increased apoptosis of the epithelial cells. LIF expression also induces phosphorylation of Stat3, the main factor signaling initiation of apoptosis, thereby suggesting that LIF is upstream of Stat3 during the early stages of involution of the mouse mammary gland [1].

Ling Zhao and colleagues (National Institutes of Health/National Institute of Diabetes and Digestive and Kidney Diseases, Bethesda, MD, USA) reported on the role of the IL-6 family of cytokines in mammary gland development. They tested the hypothesis that pathways activated by these cytokines are necessary for activation of Stat3 and p42/44 mitogen-activated protein kinase (MAPK), as both of these signals are observed in the early stages of involution. gp130 is a common receptor subunit for the IL- 6 family of cytokines such as IL- 6 and LIF; therefore, the $\mathrm{NIH}$ group characterized the mammary gland phenotypes of mice lacking either IL-6, LIF, or gp130. First, they observed in wild-type mice that mRNA levels for LIF and IL- 6 are higher during involution than lactation, and that this parallels activation of Stat3 and p42/44 MAPK. Furthermore, deletion of either IL-6, LIF, or Stat3 in the mammary gland gave delayed-involution phenotypes. However, activation of Stat3 in early involution was observed in the absence of either IL-6 or LIF, suggesting that these factors are not required for Stat3 activity.

Combined with results of Schere-Levy and colleagues, these results suggest that LIF is sufficient but not required for Stat3 activation, i.e. LIF activity can be redundant. Activation of MAPK as well as the epithelial cell-death pheno- early involution was dependent on IL-6, suggesting that the cytokines of the IL- 6 family play a role in apoptotic events needed for mammary gland involution.

\section{Stem cells}

As recently reported [2], Gilbert Smith (National Institutes of Health, Bethesda, MD, USA) presented interesting data with potential relevance to the differences between the incidence of breast cancer in women who have not given birth and those who have. More specifically, he described the presence of a population of cells with stem-cell-like properties in the involuted mammary glands of parous mice (those that had given birth) that were absent in virgin animals. He showed that a postinvolution mammary gland is fundamentally different from a virgin gland (that is, one in a mouse that had never undergone pregnancy, lactation, or involution). His evidence suggests that this difference is primarily the result of the formation of an apoptosis-resistant population of epithelial cells after the first round of pregnancy-lactation-involution. These cells function as the progenitors of alveolar structures in subsequent pregnancies. Interestingly, this epithelial cell population manifests an ability to form mammary glands upon transplantation into cleared fat pads of syngeneic immunecompromised mice, forming ducts and lobular structures and giving rise to all of the cellular subtypes found in mouse mammary epithelium, including both luminal and myoepithelial cells.

Another stem-cell-related study was presented by Bryan Welm from Zena Werb's laboratory (University of California San Francisco, San Francisco, CA, USA). This poster built on his previous work in which cells expressing stem cell antigen 1 were shown to be enriched for multipotent mammary stem cells. Here, Welm and co-workers examined several transgenic models of mouse mammary tumors and found that tumors from MMTV-Wnt-1 mice contained significantly more cells positive for stem cell antigen 1 than tumors from MMTV-Neu or MMTV-polyoma middle-T mice. These data suggest that different oncogenic lesions affect the mammary stem cell compartment in different ways, and that overexpression of Wnt-1 induces an expansion of the progenitor cell population.

\section{Matrix metalloproteinases}

Zena Werb's laboratory (University of California San Francisco, San Francisco, CA, USA) presented posters on the roles of matrix metalloproteinase 14 (MMP14) (membranetype-1 MT1-MMP) in mammary gland development, and of MMP9 in mammary tumorigenesis. Using grafts of mammary glands from wild-type and MMP14-null mice under the kidney capsules of immune-compromised mice as an assay, Heidi Mananzan and colleagues found that the number and the length of mammary epithelial ducts were decreased in the absence of MMP14. In addition, cleavage of collagen type I was greatly reduced in the 
absence of MMP14 activity. These results add MMP14 to the ever-increasing list of MMPs and other factors that affect mammary gland development.

Mikala Egeblad, also from Werb's laboratory, reported an unexpected effect of MMP9 in mouse mammary tumorigenesis. Increased MMP9 expression in human cancers correlates with metastasis, suggesting a role for MMP9 in human breast cancer progression. However, in this study, targeted deletion of MMP9 in mouse mammary models of breast cancer such as MMTV-Neu and MMTV-Wnt-1 resulted in increased tumorigenesis. An understanding of the mechanisms underlying such results could shed light on the unexpectedly low success rate of MMP inhibition in clinical trials for cancer treatment [3].

Previous collaborative work between members of the Werb laboratory and of Mina Bissell's laboratory (Lawrence Berkeley National Laboratory, Berkeley, CA, USA) showed that transgenic mice overexpressing MMP3 (stromelysin-1) exhibited increased mammary tumorigenesis [4]. Additionally, treatment of nonmalignant mammary epithelial cells (Scp2) with MMP3 induced scattering and expression of mesenchymal cell markers, as well as cleavage of E-cadherin [5]. Jimmie Fata and colleagues reported that this epithelial-to-mesenchymal transition is also observed with MMP9 but not with MMP2, MMP7, or MT1-MMP. In addition, they described an assay that is currently under development to ask if $\mathrm{E}$-cadherin cleavage is necessary for epithelial-to-mesenchymal transition, and therefore for acquisition of malignancy.

\section{Epimorphin}

Epimorphin/syntaxin 2 has been reported to induce branching morphogenesis of cultured mammary epithelial cells [6]. Following up on these observations, members of the Bissell laboratory (Lawrence Berkeley National Laboratory, Berkeley, CA, USA) presented studies on the role of epimorphin in mammary gland development. Jamie Bascom described a transgenic mouse model, expressing epimorphin (with an IL-2 signal peptide for cell-surface expression) from a mammary-specific WAP (whey acidic protein) promoter. They reported that epimorphin can indeed perturb mammary gland development in vivo. The main features of the developmental phenotype include precocious lobuloalveolar development, thicker ducts, and extensive lateral branching, in addition to a delayed-involution phenotype. Bascom and colleagues also examined epimorphin expression during wild-type gland development and found its expression levels increased during ductal development as well as during pregnancy. These data support a role for epimorphin in mammary gland branching morphogenesis in vivo.

Simone Bennett and Derek Radisky from the Bissell laboratory described the structure-activity relationships of epimorphin. By using mutational analysis and molecular modeling to compare epimorphin/syntaxin 2 to the wellstudied and highly homologous syntaxin $1 \mathrm{~A}$, they identified the helical domain in which the activity of epimorphin resides and showed that mutagenesis of a homologous three-helix bundle affects the activity of the molecule and its presentation at the cell surface.

\section{Tumor suppressors, oncogenes, and signaling pathways}

Exciting data were presented about additional roles for the p53 tumor suppressor, the identification of novel downstream targets of c-Myc and further analysis of many of the signaling pathways relevant to mammary development and tumorigenesis, including the phosphoinositide 3-kinase (PI3K) pathway, epidermal growth factor receptor (EGFR) signaling, molecules signaling cell-cell interaction such as cadherins, and many others. Here we present a selected set of breast-cancer-relevant studies relating to tumor suppressors, oncogenes, and signaling pathways.

\section{p53}

Martha Stampfer, Paul Yaswen, and colleagues (Lawrence Berkeley National Laboratory, Berkeley, CA, USA), in collaboration with Thea Tlsty (University of California San Francisco, San Francisco, CA, USA), presented their work on the effect of p53 on senescence and immortalization of mammary epithelial cells in vitro. Cell immortalization is a necessary step in tumorigenesis. Long-term collaborative work of Stampfer, Tlsty, and Yaswen has resulted in the hypothesis that there is an $\mathrm{M} 1 /$ senescence/selection barrier, followed by a second barrier, called agonescence, which human mammary epithelial cells (HMECs) need to overcome in order to become immortal. It has been shown that abrogation of the $\mathrm{Rb}$ pathway can remove the first block, and the reacquisition of telomerase activity can overcome the second block, to allow immortalization. Here they investigated the role of the p53 pathway in agonescent and postagonescent HMECs. They reported that if agonescence is approached with nonfunctional p53, then crisis, rather than agonescence, occurs. In addition, inactivating p53-dependent cell-cycle checkpoints results in a similar crisis phenotype in these HMECs. These data led Stampfer and colleagues to suggest that most cultured cell lines arrest at the first barrier of senescence and that crisis does not occur when the p53 pathway is functional. In cells that have overcome both senescence and agonescence by chemical carcinogen treatment, p53 activity in the cells slows down the process of telomerase reactivation and acquisition of uniform cell growth, both of which are required for cell immortalization. Since most human cancers are p53-deficient, the results of Stampfer and colleagues with HMECs in vitro suggest new roles for p53 in tumorigenesis, including the potential for telomerase reactivation. 
Another role for p53 in tumorigenesis was explored by Wilma Lingle (Mayo Clinic, Rochester, MN, USA) and colleagues, who investigated the effects of various p53 mutations on centrosome structure and function. Centrosome amplification has been proposed to play a role in chromosomal instability, aneuploidy, and tumor progression. Building on previous work which showed that tumors lacking functional p53 have a significantly greater microtubule nucleation capacity than tumors with wild-type p53, Lingle and her co-workers studied the effects of two tumorderived p53 mutants in cultured breast epithelial cells. They showed that the frequency of multipolar mitoses and the in vivo microtubule regrowth rate depend on the specific p53 mutation, indicating that centrosomal homeostasis is differentially sensitive to distinct p53 mutations.

\section{c-Myc}

c-Myc has been extensively studied for its roles in proliferation, differentiation, and apoptosis, and many putative downstream targets have been identified. Despite this, many of the transcriptional target genes that act as effectors of c-Myc-induced phenotypes have yet to be identified. Alexander Swarbrick and co-workers (Garvan Institute of Medical Research, Sydney, Australia) reported that Id1 may be a critical target of c-Myc in breast cancer cells. They demonstrated this by studying the effects of both inducible overexpression and loss of c-Myc function on the expression of Id1 in the human breast carcinoma cell lines T47D and MCF-7, and in the mouse mammary epithelial cell line HC11. Id, a helix-loop-helix transcription factor that has recently been implicated in mammary epithelial cell proliferation, differentiation, and tumorigenesis, is rapidly induced upon c-Myc overexpression. Cells pretreated with antisense oligonucleotides to Id1 failed to proliferate after c-myc induction. These experiments show that Id is a downstream target of c-Myc in breast cancer cell lines and may be an important mediator of c-Myc phenotypes in breast cells.

\section{Oncogenic signaling}

Wan-Nan Chen and Brian Thrall (Pacific Northwest National Laboratory, Richland, WA, USA) presented their findings on the relationship between the EGFR autocrine signaling and tumor necrosis factor alpha (TNF- $\alpha$ ) activity in HMECs. TNF- $\alpha$ is commonly associated with the inhibition of proliferation in mammary cancer cells, whereas EGFR transmits proliferation signals. Using normal HMECs (HMEC184A1), Chen and Thrall showed that low concentrations of TNF- $\alpha$ induce cell proliferation and migration in this model. These effects were sensitive to inhibitors of EGFR. TNF- $\alpha$ treatment stimulated the secretion of MMP9, and treatment of the cells with an MMP inhibitor blocked TNF- $\alpha$-induced cell migration.

Dinah Levy and colleagues from the Bissell laboratory
USA) reported the effects of isoforms of c/EBP $\beta$ (CCAAT enhancer binding protein beta) in epithelial-to-mesenchymal transition in mouse mammary epithelial cells. This protein is essential for normal mammary gland morphogenesis. Two antagonistic isoforms, liver-enriched inhibitory protein (LIP) and liver-enriched activator protein (LAP), are expressed from $c / E B P \beta$. The ratio of these isoforms changes throughout development and is altered in breast cancer. Normal mammary epithelial cells respond to laminin-rich basement membrane by increasing the LAP/LIP ratio, a response that is lost in tumor cells. Levy addressed the functional consequence of altering the LAP/LIP ratio in cultured malignant mouse mammary epithelial cells. Overexpression of LAP in these tumor cells resulted in a partial reversion of the malignant phenotype, with reorganization of tight junctions and acquisition of epithelial-like morphology.

The role of cadherins in an in vivo mouse model of metastasis was investigated by Vladimir Krutovskikh and coworkers (International Agency for Research on Cancer, Lyon, France). A cadherin-negative highly metastatic human breast cancer cell line, SKBR3, was transfected with constructs expressing either $\mathrm{E}$ - or $\mathrm{N}$-cadherin. E-cadherin expression reduced the number of metastatic nodules detected in the axillary lymphatic glands. $\mathrm{N}$-cadherin expression increased both the number and size of metastases in this model. Interestingly, a synergistic effect on metastasis was observed when E- and $\mathrm{N}$-cadherin-expressing cells were coinoculated.

The role of the PI3K/Akt-signaling pathway in mammary morphogenesis was a common thread that ran through a number of posters. Akt is recruited to the membrane by binding to phosphatidylinositol 3,4,5-phosphate moieties generated by $\mathrm{PI} 3 \mathrm{~K}$, and, once recruited, it is activated by phosphorylation at Thr308 and Ser473. The Thr308 kinase has been identified as PDK1, but as yet the identity of the kinase responsible for Ser473 phosphorylation has remained elusive. Chohren Partovian (Dartmouth Medical School, Hanover, NH, USA) presented some interesting data suggesting that this PDK2 activity is actually protein kinase $\mathrm{C}$ alpha. Protein kinase $\mathrm{C}$ alpha phosphorylates Ser473 in vitro, and siRNA-mediated down-regulation of this enzyme in endothelial cells attenuated the activity of Akt in response to insulin-like growth factor- 1 .

\section{Models of mammary gland morphogenesis and tumorigenesis in extracellular matrix in vitro}

A plethora of presentations using three-dimensional extracellular matrix models of mammary acini pointed to the beginning of a new era in breast cancer research. Pioneered by Mina Bissell (Lawrence Berkeley National Laboratory, Berkeley, CA, USA) and Ole Petersen (Panum Institute, Copenhagen, Denmark) about a decade ago [7], three-dimensional cultures of mammary epithelial cells are 
now gaining widespread appreciation as physiologically relevant models for in vitro studies. The Bissell and Petersen laboratories described the use of laminin-rich basement membrane (Matrigel) cultures of nonmalignant and malignant mammary epithelial cells of the HMT-3522 tumor progression series [4,7]. The growth of the nonmalignant cells was arrested after they had formed polarized acinar structures resembling mammary acini in vivo. Conversely, the growth of tumorigenic counterparts of these cells failed to be arrested in basement membrane cultures and the cells formed disorganized, nonpolarized colonies.

\section{MCF10A mammary acini}

Using similar paradigms but a different cell model, the members of Joan Brugge's laboratory (Harvard Medical School, Boston, MA, USA) presented an excellent series of posters and talks on their recent work on the morphogenesis of MCF10A mammary acini in vitro [8]. When grown in three-dimensional culture, MCF10A cells form polarized acini with a hollow lumen that is formed by the death of luminal cells. Loss of survival signals from extracellular matrix is a critical regulator of luminal cell apoptosis. Mauricio Reginato, Kenna Mills, and colleagues working in Brugge's laboratory investigated the mechanisms involved in the clearance of these cells to form a hollow lumen. Using a candidate-gene approach, they identified two proapoptotic factors, Bim and TRAIL, that are up-regulated at different times during morphogenesis. They showed that Bim, a proapoptotic member of the Bcl2 family, is highly up-regulated upon cell detachment from the extracellular matrix. Overexpression of oncogenes such as $E R B B 2$ can inhibit this anoikis, and overexpression of EGFR blocks the induction of Bim. Activated alleles of Ras, Raf, and MEK also block Bim up-regulation, implicating this pathway downstream of EGFR. Inhibition of Bim using siRNA delays the apoptotic clearance of the lumen. The expression of Bim is suppressed by signals from the extracellular matrix via $\beta_{1}$-integrin and MEK. Interestingly, Bim was expressed throughout the acinar structure, while only the luminal cells underwent apoptosis; this observation suggests that these cells are differently sensitive to the death signal. Inhibition of TRAIL at this time point had no effect, but at later time points, dominant-negative TRAIL blocked the apoptosis of cells that repopulate the lumen later in morphogenesis. Mills presented a model in which Bim and TRAIL mediate two distinct phases of apoptosis during luminal morphogenesis.

Carolyn Wrobel and colleagues from the Brugge laboratory probed the effects of the colony-stimulating-factor- 1 receptor (CSF-1R) signaling pathway in the MCF10A acinar morphogenesis model. In cells transfected with this receptor tyrosine kinase, treatment with its ligand, CSF1, induced normal morphogenesis, suggesting that the CSF-1/CSF-1R pathway can substitute for the interaction between epidermal growth factor (EGF) and EGFR in promoting morphogenesis. Overactivation of this pathway, either with high levels of ligand or a constitutively active mutant of the CSF-1R, induced hyperproliferation, disrupted morphogenesis, and prevented the formation of a hollow lumen. The use of pharmacological inhibitors suggested that the effects of CSF1 in this system require the activities of both Src and matrix metalloproteinases.

Jayanta Debnath, also from the Brugge laboratory, investigated the role of the PI3K/Akt signaling pathway in the formation of the acinar lumen. Using phosphospecific antibodies, they detected active Akt only in the polarized outer cells of these acini. Expression of a constitutively active Akt throughout the acinus resulted in large, irregular, apolar acini. Although all cells were expressing active Akt, significant luminal apoptosis still occurred. Akt could cooperate with proliferative oncogenes such as cyclin D1 to promote morphogenesis independently of EGF. Inhibition of mTOR (mammalian target of rapamycin), a downstream effector of the PI3K/Akt pathway, blocked the effects of Akt on morphogenesis and oncogene-driven proliferation.

\section{HMT-3522 mammary acini}

Johanne Le Beyec and Sun-Young Moonlee, from the Bissell laboratory, reported exciting findings on the role of alteration of cell shape on histone acetylation in nonmalignant breast epithelial cells of the HMT-3522 series. When grown in laminin-rich basement membrane, nonmalignant breast epithelial cells underwent growth arrest and formed polarized acini. Le Beyec and colleagues reported that this differentiation was accompanied by a global deacetylation of histone H4. They went on to show that this reduction is not related either to growth arrest or to signal transduction pathways activated by the extracellular matrix. Acini cultured in basement membrane constituents differed from monolayer cultures at multiple levels, including extracellular-matrix-cell interactions, cell-cell interactions, and changes in cell shape. The authors explored the effect of cell shape alterations either by removing EGF from monolayer cultures or by growing cells on polyHEMA - an inert substrate that allows cell rounding without the addition of any extracellular matrix component. In both cases, a reduction in histone $\mathrm{H} 4$ acetylation levels was observed. This cell-rounding effect was shown to be associated with actin microfilament assembly, since disrupting them in monolayer cultures, using cytochalasin D, reversibly decreased acetylated histone H4 levels. Cumulatively, these results suggest mechanisms by which breast tissue may achieve specificity of gene expression and nuclear organization.

Collaborators from the laboratories of Bissell, Petersen, and Weaver described the isolation and characterization of three additional cell lines with phenotypes intermediate between the nontumorigenic and tumorigenic counterparts 
of the HMT-3522 mammary epithelial cell series. The newly isolated 'premalignant' cell lines form disorganized colonies in basement membrane cultures but are neither invasive nor tumorigenic. The ability to study the interrelationships between polarity, invasiveness, and tumorigenicity in a series of cell lines of identical genetic origin provides tools for the identification of genes that contribute to such phenotypes. Aylin Rizki, Valerie Weaver, and colleagues reported on global studies of the genome and gene expression profiles of the nonmalignant, premalignant, and malignant members of this tumor progression series and proposed genes whose function may be relevant to the in vivo loss of polarity and invasiveness of mammary tumors.

Using the HMT-3522 model, Gabriela Rozenberg and co-workers (University of Pennsylvania, Philadelphia, PA, USA) found that expression of both fibronectin and its receptor, $\alpha_{5} \beta_{1}$ integrin, correlated with the malignant phenotype. Interestingly, both of these factors are down-regulated upon pharmacological reversion of malignancy in these cells. These workers found that expression of wildtype $\alpha_{5}$ integrin disrupted the polarity of the nonmalignant cells if fibronectin was provided as a substrate in the basement membrane cultures and that a dominant negative $\alpha_{5}$ integrin could repress the invasiveness and restore the polarity of the malignant cells. These findings provide additional insight into the role of fibronectin-integrin interactions in the regulation of breast tissue function.

\section{Other basement membrane models}

Few good models exist for the study of the endogenous estrogen receptor in cultured, functionally normal mammary epithelial cells. Virginia Novaro and colleagues from the Bissell laboratory showed that culturing such cells in laminin-rich basement membrane could maintain functional expression of estrogen receptor alpha (ER- $\alpha$ ), which is usually lost when cells are cultured on two-dimensional plastic substrata. They showed that this effect is elicited by two components of the extracellular matrix: collagen type IV and laminin 1 . The effects of collagen type IV are mediated via its interaction with $\alpha_{2}$ and $\beta_{1}$ integrins, while regulation of ER- $\alpha$ via laminin 1 is mediated by $\alpha_{2}, \alpha_{6}$, and $\beta_{1}$ integrins. These data provide further evidence for the role of the extracellular matrix in maintaining the form and function of the mammary gland.

Loss of p53 function is a common feature of breast and other cancers. Victoria Seewaldt and colleagues (Duke University, Durham, NC, USA) investigated the consequences of acute suppression of p53 in normal, nonimmortalized HMECs in vitro. In these authors' model, the mammary cells proliferated in reconstituted basement membrane for 6 days and formed growth-arrested acini by day 7. Suppression of p53 function, by the use of either antisense oligonucleotides or retrovirally expressed E6 apoptosis was induced by the laminin component of reconstituted basement membrane and was inhibited by antibodies that block the function of either $\alpha_{3}$ or $\beta_{1}$ integrin [9].

Adhesion to the extracellular matrix is required for the survival of epithelial cells. Andrew Gilmore and colleagues (University of Manchester, Manchester, UK) investigated the effects of blocking this adhesion on the localization of the proapoptotic Bcl2-family member Bax. Bax is typically localized in the cytoplasm, but apoptotic stimuli promote its translocation to the mitochondrial membrane. A subsequent reorganization of Bax into clusters at the mitochondrial membrane stimulates the release of cytochrome $c$. When mammary epithelial cells were detached from extracellular matrix and cultured on polyHEMA (thereby blocking the activation of integrins), Bax translocated to the mitochondrial membrane within 15 minutes. Formation of supramolecular Bax clusters was a later event that correlated with cytochrome $c$ release.

\section{Global strategies for understanding breast cancer}

In the special-interest group 'Systems Biology and the Cell: Are There Simple Rules Governing Complexity?', Therese Sorlie described her work on the subclassification of breast tumors by identifying a 'molecular portrait' of various tumor types and the correlation of these expression patterns with clinical parameters such as survival and the likelihood of metastasis. Using a hierarchical clustering approach, Sorlie and colleagues stratified their samples into five broad groups: basal-cell-like, ERBB2-positive, normal-breast-like, luminal subtype $A$, and luminal subtype $B$. Both luminal subtypes were positive for expression of ER- $\alpha$ but, interestingly, these groups of ER- $\alpha$-positive tumors had distinct clinical outcomes. Tumors of the luminal subtype A class were associated with the longest survival, whereas tumors of both the ERBB2 ${ }^{+}$and basal subtypes had the poorest prognosis [10]. Significantly, analysis of other breast tumor microarray datasets $[11,12]$ using these molecular signatures extended the validity of these findings and provided additional information. Both the ERBB2 ${ }^{+}$and basal subtypes had significantly more metastases than the luminal $A$ subtype. This increase in metastases correlated strongly with both ER- $\alpha$ negativity and mutations in TP53 in the ERBB2 $^{+}$and basal subtypes. These data can be accessed via the Stanford Breast Cancer Genomics Consortium (http://genome-www.stanford.edu/breast_cancer/).

An international team comprising three groups (Rigel Inc, South San Francisco, CA, USA; University of Alberta, Edmonton, Canada; and JJPRD, Beerse, Belgium) reported a new systematic approach for the identification of small protein sequences that can inhibit the growth of tumor cells. Yasumichi Hitoshi and colleagues used a retroviral library of random 20-mer peptides fused to green fluorescent protein to screen for growth-inhibiting pep- 
tides. They described two peptides identified in this screen that induce arrest of the cell cycle at G0/G1 or $\mathrm{G} 2 / \mathrm{M}$ and are localized to the cell membrane. They went on to show that the cell-cycle-arrest phenotype is independent of the origin of the cell line (they tested cell lines from breast, lung, cervical, and colon cancer) and is independent of p53 function. They are currently identifying the binding partners of these peptides in the cell in an effort to identify the signaling pathways that are inhibited by them. Global approaches such as this are likely to yield promising drug targets for breast cancer therapy.

\section{Conclusion}

The abstracts for each talk and poster presentation have been published as a supplement to the Nov 2002 issue of Molecular Biology of the Cell [13] and are freely searchable on line (http://www.ascb.org/meetings/ am2002/itinerarystart.htm).

In summary, this was a hugely enjoyable, varied, and informative meeting and we look forward to the 43rd annual meeting of the ASCB, which will again take place in San Francisco, from 13 to 17 December 2003.

\section{Competing interests}

None declared.

\section{References}

1. Schere-Levy C, Buggiano V, Quaglino A, Gattelli A, Cirio MC, Piazzon I, Vanzulli S, Kordon EC: Leukemia inhibitory factor induces apoptosis of the mammary epithelial cells and participates in mouse mammary gland involution. Exp Cell Res 2003, 282:35-47.

2. Wagner KU, Boulanger CA, Henry MD, Sgagias M, Hennighausen L, Smith GH: An adjunct mammary epithelial cell population in parous females: its role in functional adaptation and tissue renewal. Development 2002, 129:1377-1386.

3. Overall CM, Lopez-Otin C: Strategies for MMP inhibition in cancer: innovations for the post-trial era. Nat Rev Cancer 2002, 2:657-672.

4. Sternlicht MD, Lochter A, Sympson CJ, Huey B, Rougier JP, Gray JW, Pinkel D, Bissell MJ, Werb Z: The stromal proteinase MMP3/stromelysin-1 promotes mammary carcinogenesis. Cell 1999, 98:137-146.

5. Lochter A, Galosy S, Muschler J, Freedman N, Werb Z, Bissell $\mathrm{MJ}$ : Matrix metalloproteinase stromelysin-1 triggers a cascade of molecular alterations that leads to stable epithelial-to-mesenchymal conversion and a premalignant phenotype in mammary epithelial cells. J Cell Biol 1997, 139:1861-1872.

6. Hirai Y, Lochter A, Galosy S, Koshida S, Niwa S, Bissell MJ: Epimorphin functions as a key morphoregulator for mammary epithelial cells. J Cell Biol 1998, 140:159-169.

7. Petersen OW, Ronnov-Jessen L, Howlett AR, Bissell MJ: Interaction with basement membrane serves to rapidly distinguish growth and differentiation pattern of normal and malignant human breast epithelial cells. Proc Natl Acad Sci USA 1992, 89:9064-9068.

8. Debnath J, Mills KR, Collins NL, Reginato MJ, Muthuswamy SK Brugge JS: The role of apoptosis in creating and maintaining luminal space within normal and oncogene-expressing mammary acini. Cell 2002, 111:29-40.

9. Seewaldt VL, Mrozek K, Sigle R, Dietze EC, Heine K, Hockenbery DM, Hobbs KB, Caldwell LE: Suppression of p53 function in normal human mammary epithelial cells increases sensitivity to extracellular matrix-induced apoptosis. J Cell Biol 2001, 155:471-486.
10. Sorlie $T$, Perou CM, Tibshirani R, Aas $T$, Geisler $S$, Johnsen $H$, Hastie T, Eisen MB, van de Rijn M, Jeffrey SS, Thorsen T, Quist H, Matese JC, Brown PO, Botstein D, Eystein Lonning P, BorresenDale AL: Gene expression patterns of breast carcinomas distinguish tumor subclasses with clinical implications. Proc Nat/ Acad Sci USA 2001, 98:10869-10874.

11. West $M$, Blanchette $C$, Dressman $H$, Huang $E$, Ishida S, Spang $R$, Zuzan H, Olson JA, Jr., Marks JR, Nevins JR: Predicting the clinical status of human breast cancer by using gene expression profiles. Proc Natl Acad Sci USA 2001, 98:11462-11467.

12. Van't Veer $\mathrm{LJ}$, De Jong D: The microarray way to tailored cancer treatment. Nat Med 2002, 8:13-14.

13. Abstracts of the 42nd American Society for Cell Biology Annual Meeting. 14-18 December 2002, San Francisco, California, USA. Mol Biol Cell 2002, 13(suppl):1a-553a.

\section{Correspondence}

Aylin Rizki, Life Sciences Division, Lawrence Berkeley National Laboratory, Mailstop 83-101, 1 Cyclotron Road, Berkeley, CA 94720, USA. Tel: +1 510486 4368; fax: +1 510486 5586; e-mail: arizki@lbl.gov 